\title{
Single-Mode Polymer Embedded Waveguide for Visible Wavelength using SU-8
}

\author{
Junji Sakamoto $^{1,2^{*}}$, Toshikazu Hashimoto ${ }^{2}$, Hiroaki Kawata ${ }^{1}$, and Yoshihiko Hirai ${ }^{1}$ \\ ${ }^{1}$ Department of Physics and Electronics Engineering, Graduate School of Engineering, \\ Osaka Prefecture University, 1-1 Gakuen-cho, Naka-ku, Sakai, Osaka 599-8531, Japan \\ ${ }^{2}$ NTT Device Technology Labs, NTT Corporation, \\ 3-1 Morinosato-Wakamiya, Atsugi, Kanagawa 243-0198, Japan \\ *junji.sakamoto.nx@hco.ntt.co.jp
}

\begin{abstract}
We investigated the optical characteristics of a single-mode polymer waveguide in the visible wavelength region, including the blue range. We fabricated two types of polymer waveguide by using photolithography and UV-nanoimprint lithography. The polymer waveguides had an over and under cladding, and the refractive index difference between core and cladding was $0.8 \%$ at a wavelength of $633 \mathrm{~nm}$. The propagation loss of the fabricated waveguide was about 0.8 to $4.4 \mathrm{~dB} / \mathrm{cm}$ at wavelengths from 638 to $465 \mathrm{~nm}$. The waveguide worked as a single-mode one over the whole visible region.
\end{abstract}

Keywords: Polymer waveguide, Single-mode, Visible light, SU-8, UV-nanoimprint, RGB coupler

\section{Introduction}

There is growing interest in compact red-green-blue (RGB) light sources for glass displays and mobile projectors [1-3]. Here, a waveguide RGB coupler is needed for reducing the size of the light source through the use of integration [4-8]. The couplers are fabricated using silica planar lightwave circuit (PLC) technology, which is used in optical communications $[9,10]$. Polymer waveguides are potentially cost-effective because their fabrication process is simpler than that of silica-waveguide RGB couplers.

Many polymer optical waveguides have been reported for optical communications devices [11-17]. Since infrared light having low fiber propagation loss is used in these applications, the reported characteristics of the waveguides have been limited to a wavelength range from about 800 to $1550 \mathrm{~nm}$. As far as we know, although there are a few reports in the field of biosensing in the visible wavelength range, these only cover red wavelengths [18-20]. Thus, little is known about the characteristics of polymer waveguides in the whole visible wavelength range. For this reason, we decided to fabricate a single-mode polymer waveguide and measure its optical characteristics throughout the visible wavelength range.

\section{Structure and fabrication}

We examined two kinds of polymer as a core material, SU-8 (from Microchem Corporation) and CELVENUS (from DAISEL Corporation), because of their ease of patterning and good transparency to visible light. SU-8 can be patterned by photolithography, while CELVENUS can be patterned using UV-nanoimprint lithography (NIL). Figure 1 shows the structure of the polymeric embedded waveguide. Since patterning of SU-8 is limited to a few micrometers, an embedded waveguide having a small refractive index difference $(\Delta)$ is used so that single-mode operation can be maintained even at this size.

$\mathrm{SiO}_{2}$ on $\mathrm{Si}$ substrate was used as the under cladding for obtaining a stable refractive index. An epoxy-based polymer with a controllable refractive index was used as the over cladding. The left side of Fig. 1 shows the calculated fundamental mode at a wavelength of $638 \mathrm{~nm}$. The core height and width were each $2.0 \mu \mathrm{m}$. The over and under cladding thicknesses were each $15 \mu \mathrm{m}$, and $\Delta$ was 
$0.8 \%$. To obtain a structure close to that of the reference [6], the parameters were set to have similar values as the reference. Since the refractive index distribution was asymmetric, the center of the mode field shifted a bit to above the center of the core.

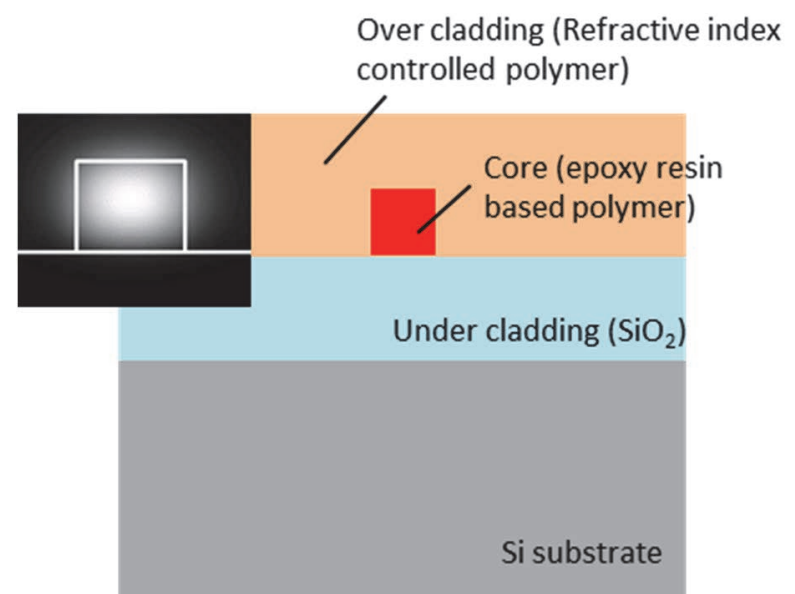

Fig. 1. Polymer waveguide structure and calculated fundamental mode.
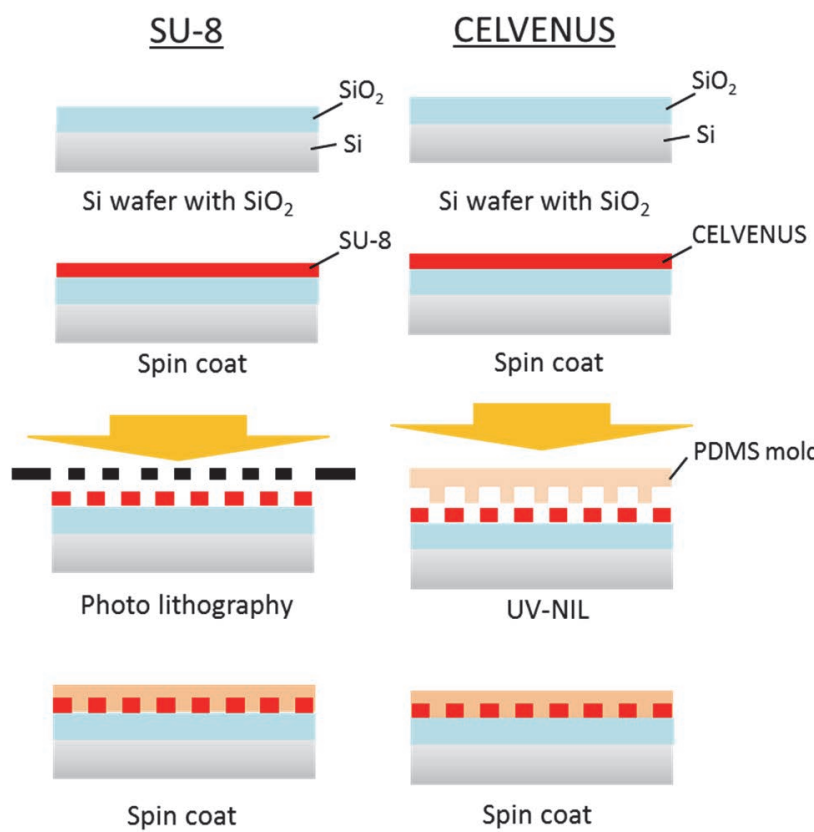

Spin coat
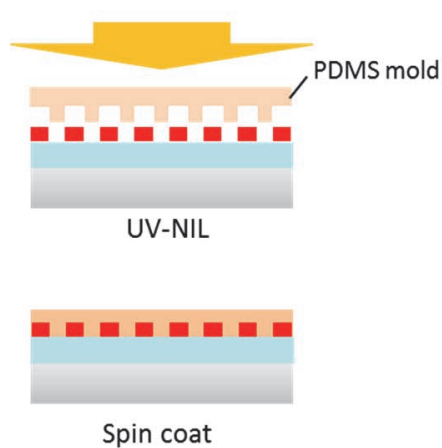

Fig. 2. Fabrication process.

Figure 2 shows the fabrication process. SU-8 was spin coated on a $\mathrm{Si}$ wafer with $\mathrm{SiO}_{2}$ to a thickness of $2.0 \mu \mathrm{m}$. To obtain a thin film, the SU-8 was diluted with SU-8 thinner. After prebaking (for $300 \mathrm{~s}$ at $65{ }^{\circ} \mathrm{C}$ and for $900 \mathrm{~s}$ at 95 ${ }^{\circ} \mathrm{C}$ ), the SU-8 film on $\mathrm{SiO}_{2}$ was patterned by i-line photolithography. The SU-8 core pattern was obtained after post baking $\left(60 \mathrm{~s}\right.$ at $65{ }^{\circ} \mathrm{C}$ and $120 \mathrm{~s}$ at $95{ }^{\circ} \mathrm{C}$ ) and development. Finally, an epoxy-based polymer whose refractive index was adjusted so that $\Delta$ was $0.8 \%$ at a wavelength of 633 $\mathrm{nm}$ was spin-coated. The CELVENUS core pattern was fabricated by using UV-NIL. Using the same photomask was used in the SU-8 waveguide fabrication process, an $\mathrm{SiO}_{2}$ pattern matching that of the SU-8 pattern was fabricated by dry etching. The fabricated $\mathrm{SiO}_{2}$ pattern was used as a master mold to prepare a PDMS replica mold. A CELVENUS core pattern was then fabricated by using UV-NIL with the PDMS replica mold. Finally, an epoxy-based polymer whose refractive index was such that $\Delta$ was $0.8 \%$ at a wavelength of $633 \mathrm{~nm}$ was spin-coated. The core pattern using CELVENUS before spin-coated an over cladding is shown in Fig. 3. The imprinted pattern was successfully obtained.

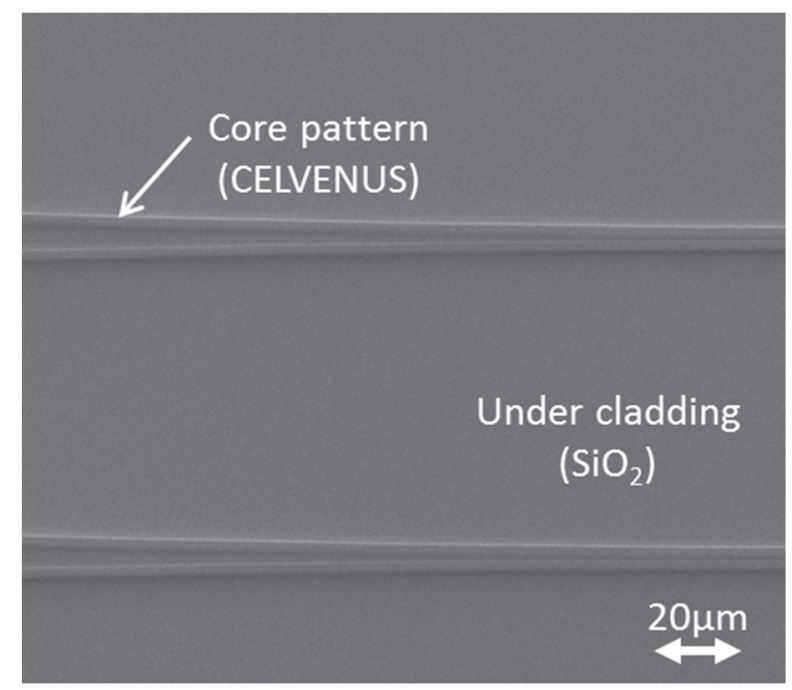

Fig. 3. SEM image of the core pattern using CELVENUS before spin-coated an over cladding.

\section{Evaluation of fabricated polymeric optical waveguides}

We investigated the characteristics of the fabricated waveguides as well as the optical circuit performance to confirm their feasibility for RGB couplers.

\subsection{Characteristics of the waveguides}

Figure 4 shows the experimental setup for measuring the optical characteristics of the waveguide. 


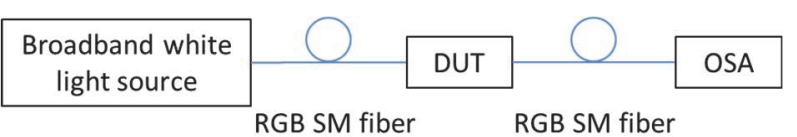

Fig. 4. Experimental setup.

The waveguide was evaluated as a single-mode fiber but with the coupling through its input/output port. A non-polarized broadband white light source and optical spectrum analyzer (OSA) were used. The core width and height were 1.7 and $2.0 \mu \mathrm{m}$.

The measured $\Delta$ between the core and over cladding is shown in Fig. 5.

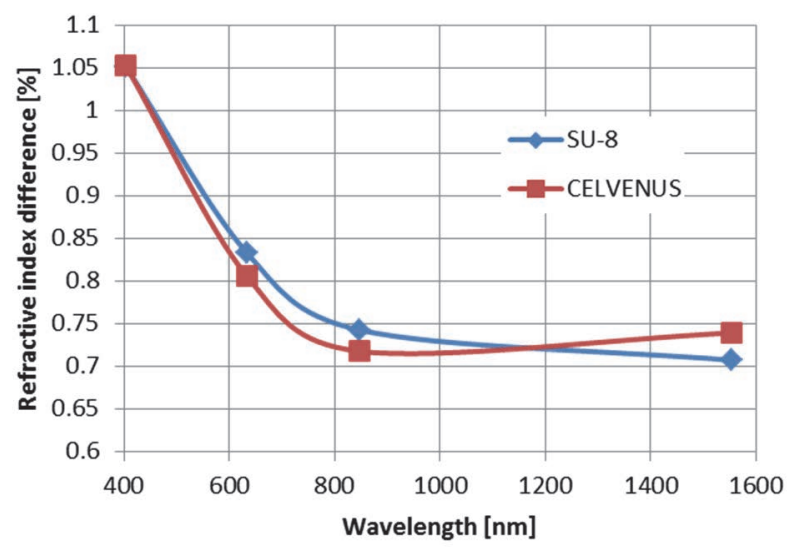

Fig. 5. Measured wavelength dispersions of $\Delta$ of SU-8 and CELVENUS waveguide.

Since the wavelength dispersions of the refractive indexes of the core and cladding materials were different, the short wavelength side $\Delta$ became large.

The relationship between the length of the straight waveguide and the transmittance is shown in Fig. 6 for wavelengths of 465, 520, and $638 \mathrm{~nm}$. The transmittance in this case included fiber coupling loss. The measurements were conducted using the cut back method. The 40-mm-long CELVENUS waveguide could not be measured because the receiver was limited in its measurement capability at the wavelength of 465 $\mathrm{nm}$. The transmittance of the waveguides changed almost linearly, except for the 40-mm-long CELVENUS waveguide at $520 \mathrm{~nm}$ as it was affected by fabrication errors such as imprinting defects or particles on the waveguide. The results indicate that the SU- 8 waveguide had propagation losses of about 4.4, 2.0, and $0.8 \mathrm{~dB} / \mathrm{cm}$ at wavelengths of 465,520 , and $638 \mathrm{~nm}$, while the CELVENUS waveguide had propagation losses of about 10.6, 5.1 , and $0.8 \mathrm{~dB} / \mathrm{cm}$ at wavelengths of
465, 520, and $638 \mathrm{~nm}$. The fiber coupling loss was about $1.5 \mathrm{~dB} /$ point at $638 \mathrm{~nm}$, which is close to the value $(1.3 \mathrm{~dB} /$ point $)$ calculated by the three-dimensional mode solver.
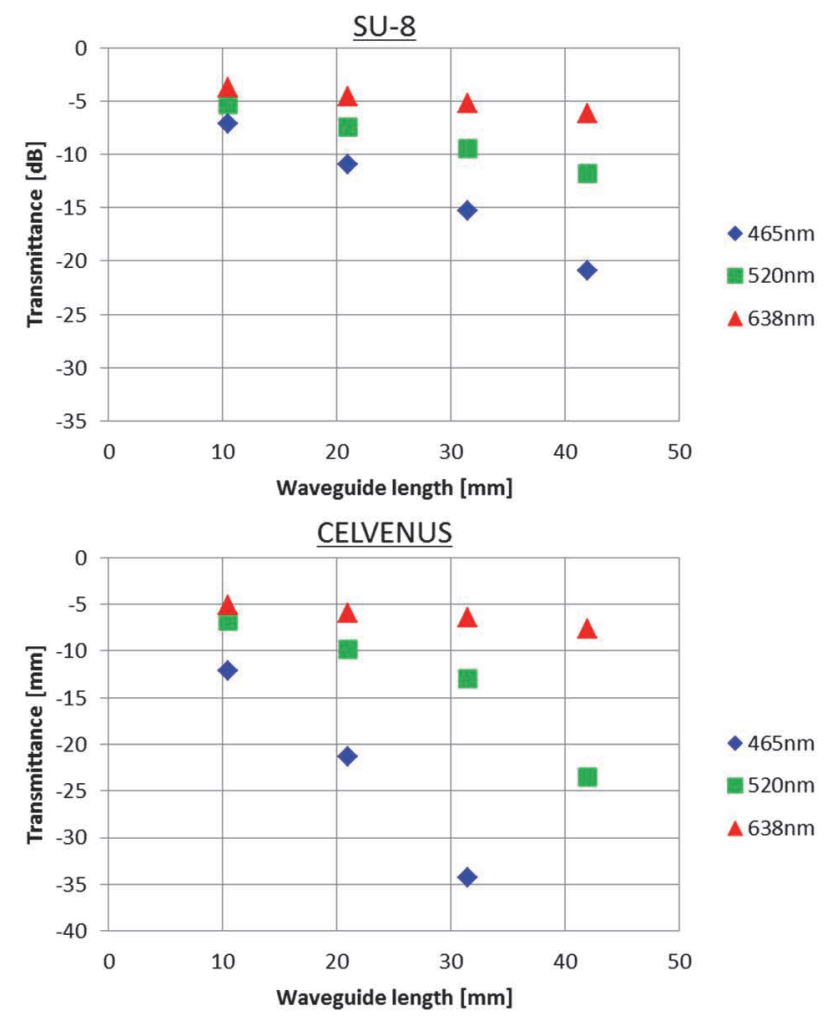

Fig. 6. Transmittances of different lengths of SU-8 and CELVENUS waveguide.

Although the transmittance of the SU-8 waveguide was better than that of the CELVENUS waveguide, we selected the SU-8 waveguide for the evaluation of wavelength coupler performance. Before the coupler evaluation, we confirmed that the SU-8 waveguide provides single mode operation even for blue by observing the output near field pattern (NFP). Figure 7 shows the NFP for wavelengths of 638,520 , and $450 \mathrm{~nm}$. One can see that the fundamental mode is emitted and the waveguide operates in single mode. Since the confinement of the waveguide is weaker at longer wavelengths, the NFP is wider at longer wavelengths. Figure 8 shows the intensity of the NFP. The NFP in the vertical direction spreads more at higher wavelengths, and overall, it has an asymmetric shape. This is due to the asymmetry of the refractive index of the over and under cladding; the measurements are in good agreement with the calculation results shown in Fig. 1. 


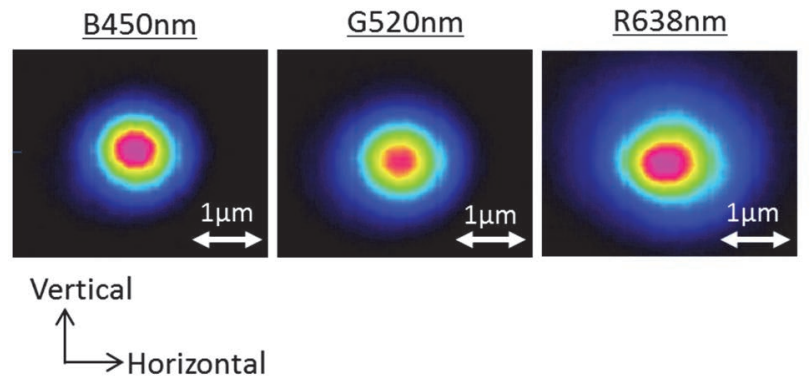

Fig. 7. NFP from SU-8 waveguide when R, G, and B light (wavelength of $638,520,450 \mathrm{~nm}$ ) was input.
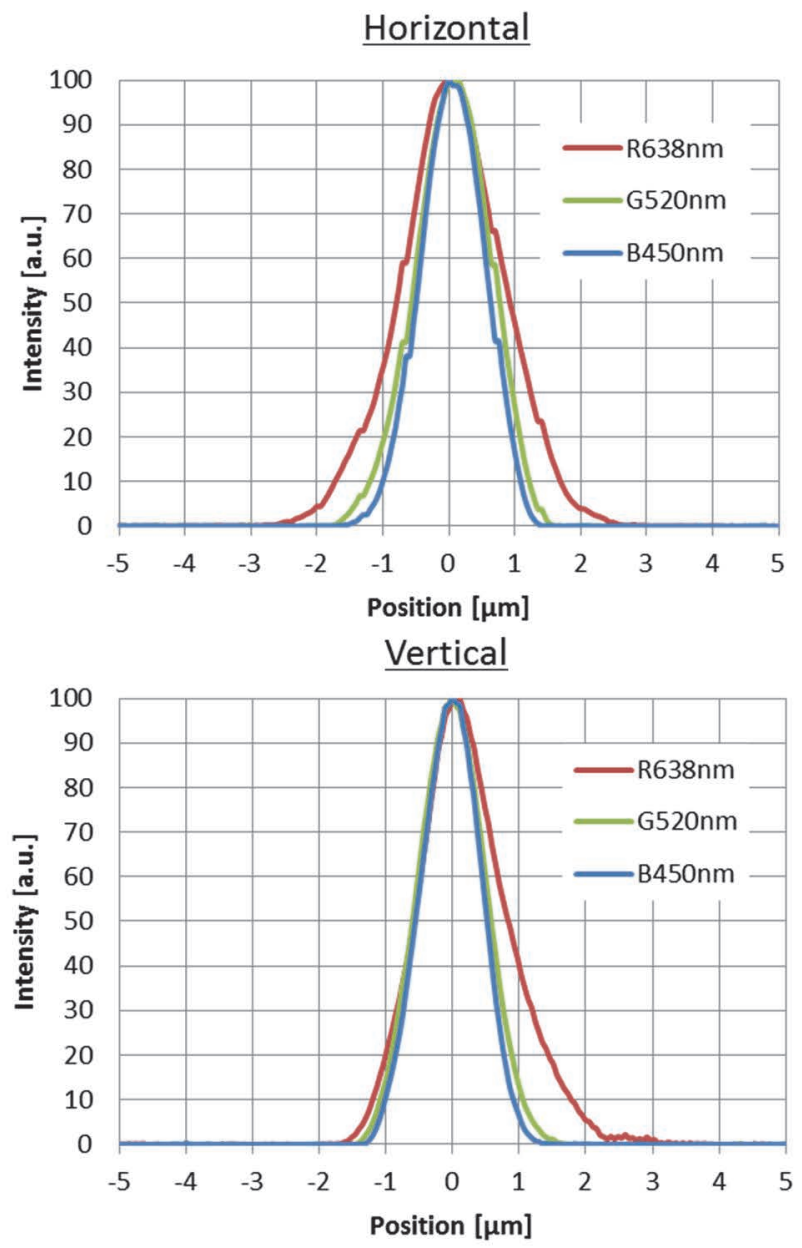

Fig. 8. Intensity of NFP at wavelengths of 638,520 , and $450 \mathrm{~nm}$.

\subsection{Characteristics of optical circuit (directional coupler)}

Next, we measured the characteristics of a directional coupler using the SU-8 waveguide. The width, gap, and length of the coupler were 2.5, 1.5, and $850 \mu \mathrm{m}$ (Fig. 9). A bending simulation using the three-dimensional beam propagation method (BPM) indicated that the minimum radius at which no bending loss occurs at a wavelength of $638 \mathrm{~nm}$ is $1.3 \mathrm{~mm}$. In the experiment, the radius of the bending part was $10 \mathrm{~mm}$, which was sufficiently large so that no bending loss would have occurred in the RGB wavelength range. The measurement results are shown in Fig. 10. The spectra are plotted without taking account of the propagation or fiber coupling loss. This was accomplished by placing a reference straight waveguide in parallel with the coupler. The coupler worked well except in the blue region; the disturbances in the blue region were caused by the poor receiver performance at low power. Although we expect that good coupling performance can be maintained in the blue region because the NFP in Fig. 7 indicates that the waveguide operates in single mode at a wavelength of $450 \mathrm{~nm}$, the transmittance of the waveguide should be improved so that the loss would be acceptable for optical circuits of several $\mathrm{mm}$ and further measurements should be made [5].

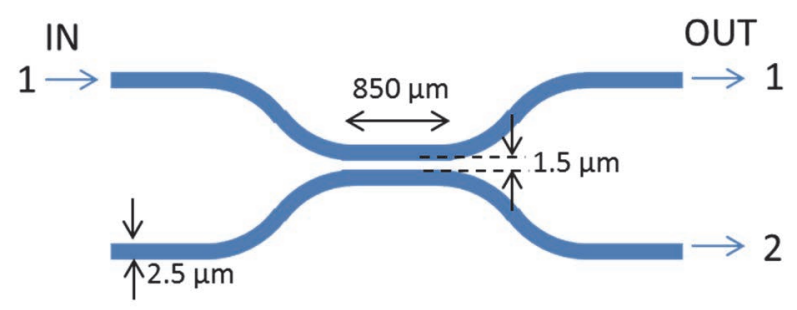

Fig. 9. Structure of measured directional coupler of SU-8 waveguide.

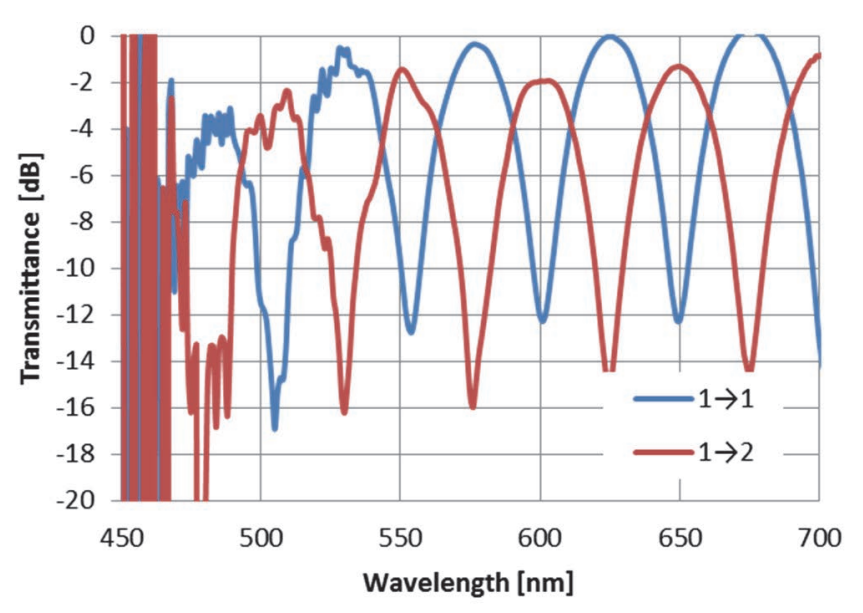

Fig. 10. Spectra of directional coupler fabricated using SU-8. A reference straight waveguide, which was placed in parallel with the coupler, eliminated the propagation and fiber coupling loss. 


\section{Conclusion}

We confirmed the feasibility of a single-mode polymer waveguide for visible light including wavelengths in the blue region. Polymer waveguides were fabricated by photolithography and UV-NIL. Although the propagation loss was still large at short wavelengths, the waveguide nonetheless operated in a single mode at RGB wavelengths. By improving the fabrication accuracy and changing the polymer material to one with high transparency in the blue region, we believe that this sort of waveguide will be able to be used as an RGB coupler.

\section{References}

1. J. Tauscher, W. O. Davis, D. Brown, M. Ellis, Y. Ma, M. E. Sherwood, D. Bowman, M. P. Helsel, S. Lee, and J. W. Coy, Proc. SPIE, 7594 (2010) 75940A.

2. J. Pan, S. Tu, C. Wang, and J. Chang, Appl. Opt., 47 (2008) 3406.

3. W. O. Davis, R. Sprague, and J. Miller, IEEE/LEOS International Conference on Optical MEMS and Nanophotonics, Germany, (2008) pp. 31.

4. J. Sakamoto, S. Katayose, K. Watanabe, M. Itoh, and T. Hashimoto, Proc. SPIE, 10126 (2017) $101260 \mathrm{M}$.

5. J. Sakamoto, T. Goh, S. Katayose, K. Watanabe, M. Itoh, and T. Hashimoto, Opt. Commun., $\mathbf{4 2 0}$ (2018) 46.

6. J. Sakamoto, T. Goh, S. Katayose, R. Kasahara, and T. Hashimoto, Opt. Commun., 433 (2019) 221.

7. A. Nakao, R. Morimoto, Y. Kato, Y. Kakinoki, K. Ogawa, and T. Katsuyama, Opt. Commun., 330
(2014) 45.

8. T. Katsuyama, A. Nakao, K. Ogawa, K. Tsujino, and K. Takahata, Proc. SPIE, 9272 (2014) 927203.

9. M. Kawachi, IEE Proc. Optoelectron., 143 (1996) 257.

10. R. Nagase, A. Himeno, M. Okuno, K. Kato, K. Yukimatsu, and M. Kawachi, J. Lightwave Technol., 12 (1994) 1631.

11. M. Wang, J. Hiltunen, S. Uusitalo, J. Puustinen, J. Lappalainen, P. Karioja, and R. Myllylä, Microelectron. Eng., 88 (2011) 175.

12. L. Eldada and L. W. Shacklette, J. Sel. Top. Quantum Electron., 6 (2000) 54.

13. K. K. Tung, W. H. Wong, and E. Y. B. Pun, Appl. Phys. A: Mater. Sci. Process., 80 (2005) 621.

14. J. S. Kim, J. W. Kang, and J. J. Kim, Jpn. J. Appl. Phys., 42 (2003) 1277.

15. N. Keil, H. H. Yao, C. Zawadzki, J. Bauer, M. Bauer, C. Dreyer, and J. Schneider, Electron. Lett., 37 (2001) 579.

16. S. Ando, J. Photopolym. Sci. Technol., 17 (2004) 219.

17. A. Mochizuki, K. Mune, R. Naitou, T. Fukuoka, and K. Tagawa, J. Photopolym. Sci. Technol., 16 (2003) 243.

18. B. Yang, L. Yang, R. Hu, Z. Sheng, D. Dai, Q. Liu, and S. He, J. Lightwave Technol., 27 (2009) 4091.

19. M. Wang, J. Hiltunen, C. Liedert, S. Pearce, M. Charlton, L. Hakalahti, P. Karioja, and R. Myllylä, Opt. Express, 20 (2012) 20309.

20. Y.-G. Zhao, W.-K. Lu, Y. Ma, S.-S. Kim, S. T. Ho, and T. J. Marks, Appl. Phys. Lett., 77 (2000) 2961. 